\title{
Learning Sustainable Locust Control Methods in Virtual Reality
}

This paper was downloaded from TechRxiv (https://www.techrxiv.org).

\section{LICENSE}

CC BY 4.0

SUBMISSION DATE / POSTED DATE

$30-01-2022$ / 10-02-2022

\section{CITATION}

Hahn, Nathan; Fuchs, Beatrice; Fortna, Max; Cobb, Elijah; Iqbal, Muhammad Zahid (2022): Learning Sustainable Locust Control Methods in Virtual Reality. TechRxiv. Preprint. https://doi.org/10.36227/techrxiv.19093160.v2

$\mathrm{DOI}$ 


\section{Learning Sustainable Locust Control Methods in Virtual Reality}

\author{
Nathan Hahn \\ George Mason University \\ Fairfax, Virginia, United States of America \\ nhahn@masonlive.gmu.edu \\ Elijah Cobb \\ Northeastern University \\ Boston, MA, USA \\ Ecobb81@gmail.com
}

\author{
Beatrice Fuchs, MA \\ University of Applied Science BFI Vienna \\ Vienna, Austria \\ beatrice.windfuchs@gmail.com \\ Muhammad Zahid Iqbal \\ University College Dublin \\ Dublin, Ireland \\ Muhammad-zahid.iqbal@ucdconnect.ie
}

\author{
Max Fortna \\ Northeastern University \\ Boston, MA, USA \\ fortna.m@northeastern.edu
}

\begin{abstract}
Invasion of locust swarms has affected the crops in many countries in Africa and Asia which is a major threat to food security. According to the Food and Agriculture Organisation (FAO), locusts have the ability to fly over $130 \mathrm{~km}$ a day and stay in the air for a long time. There are different approaches adopted in the different parts of the world to monitor and control the locust swarms to save the crops. It has been proved in various studies that technology can help in agriculture through drones, real-time data monitoring or teaching the farmers with latest tools.

Following the UN sustainability goals for food security, this research has presented a virtual reality based educational game to teach sustainable locust management strategies. Hand tracking technology in the Oculus Quest enables users to learn the ways that farmers deal with pests without using pesticides. The methods shown are not only profitable for the farmers but also free of any harm for crops in terms of food security. This game can help to motivate the adoption of these sustainable locust control strategies in broader interventions for environmental recovery.

Index Terms-ICT in agriculture, locusts swarms, virtual reality, ICT for Development
\end{abstract}

\section{INTRODUCTION}

Locusts are a group of short-horned grasshoppers. They are considered as the world's most dangerous migratory pest because of the number of locusts in the swarms and flying speed [1]. They cause destruction of crops which is a threat for food security for millions of people in the world. The locusts attacks happen due to seasonal migration due to the climatic factors such as temperature, wind and vegetation [2] [3]. As a natural phenomenon, locusts are controlled by birds, parasitic flies and spiders as they are using them as food, but these are not effective when numbers explode. In recent years, locust swarms generated significant agricultural losses in India, Pakistan and African countries that are already unstable in terms of economics and food security [4]. These outbreaks threaten to increase food shortages in a region.

Locusts are a very rich source of protein and provide an alternative livestock or human protein source [5] as food.

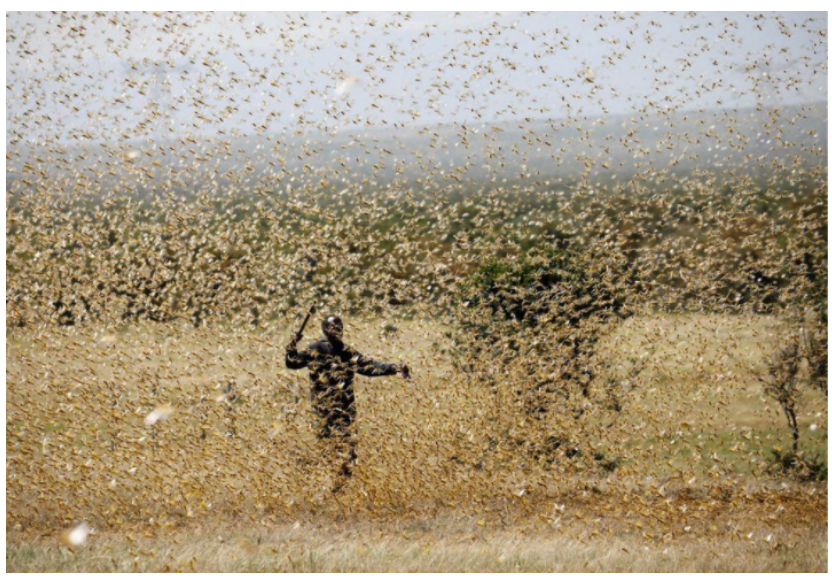

Fig. 1. Locust swarm attacking on crops

Current strategies to control the locust attacks are including early warnings and preventive control [6].

\section{RELATED WORK}

In different parts of the world, there are different strategies to control the locust. These strategies include the use of pesticides, the use of drumming sounds to scare away locusts and using locusts eating animals [7]. Locusts are used as food because they are a rich source of protein for humans and animals. Locust outbreaks are variable in many factors including geography, environmental sensitivity, availability of resources and rainfall patterns [8].

Technology has been used in different formats to control the locusts. For example, an automated Locust Management using Artificial Intelligence has been developed for drones to detect and kill the locusts real-time using pesticides [9]. In China, chickens and ducks army has been used for combating the locusts as it is a natural way of dealing with locusts [10].

There are different methods and tools adopted by Food and Agriculture Organization of United Nations like eLocust3 [11], Track Guidance System, Ultra Low Volume and drone 

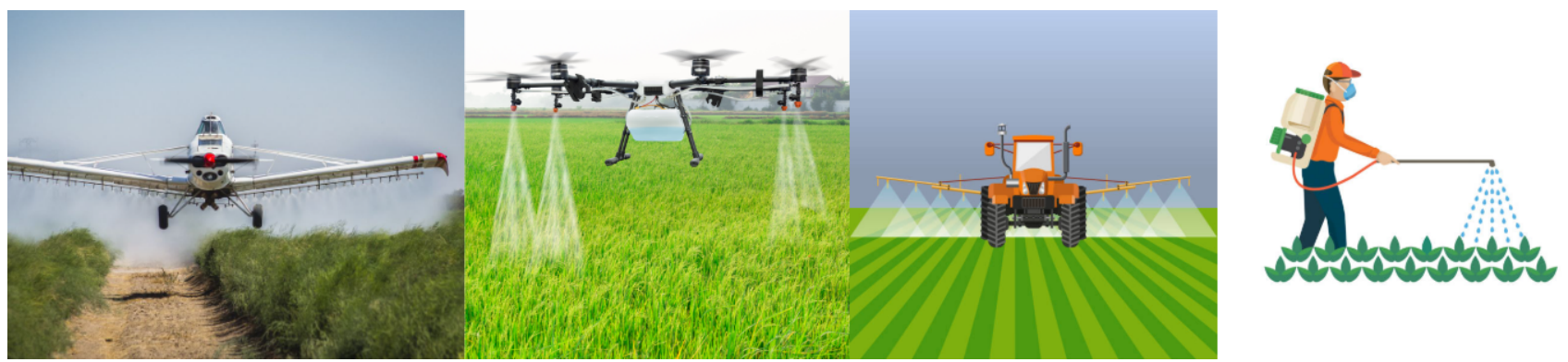

Fig. 2. Different methods of using pesticides for crops to stop the locusts attack

technologies [12] that allow locust management to bring locust under control before it does severe damage for the crops [13].

To provide support for farmers about controlling locusts, the Locust Prevention and Control DSS (LPCDSS) system (Fig.3) was introduced in China to provide real time location based data for farmers and providing information on the population of locusts in the specific area [14].

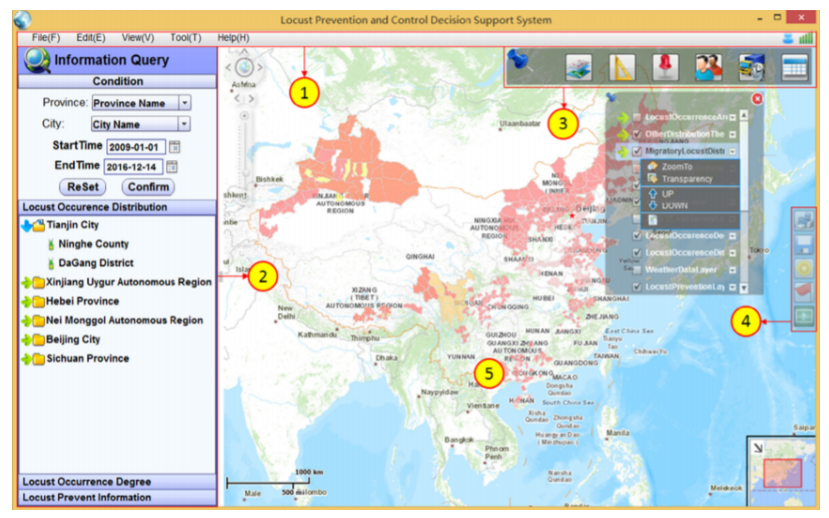

Fig. 3. Web interface of Locust Prevention and Control DSS(LPCDSS)

The Chinese duck army is trained to eat swarms of cropeating pests for regional food security. According to one source, "One duck is able to eat more than 200 locusts a day" [15]. Ducks use the locusts as a source of food and preserve the crop fields from swarm attacks.

Fig. 2 is shows different methods of pesticides used for locust control which are considered harmful for food and costly as well. With all of these different ways of controlling locusts, this research's aim is to adopt the safest ways of locust control instead of using harmful pesticides.

\section{GAME DESIGN}

Our game places players in the perspective of Natalina, a farmer who needs to defend her farm against a swarm of locusts. By using VR hand tracking technology and developing game mechanics that reflect real-world locust defense management strategies, they will be able to learn and appreciate the challenges and possibilities of sustainable locust control methods.

The interaction in this game uses the Oculus Quest's hand tracking technology instead of traditional VR hand controllers.
It is actually based on kinesthetic learning style by replacing the learning material with the virtual one in the resource constraint learning space [16].

The player has four ways in the game to combat locusts;

- Hitting the locusts with their hands to temporarily shoo them away

- Catching the locusts with a pinch action to earn money

- Starting controlled fires to deter the locusts through smoke

- Purchasing ducks which will eat the locusts

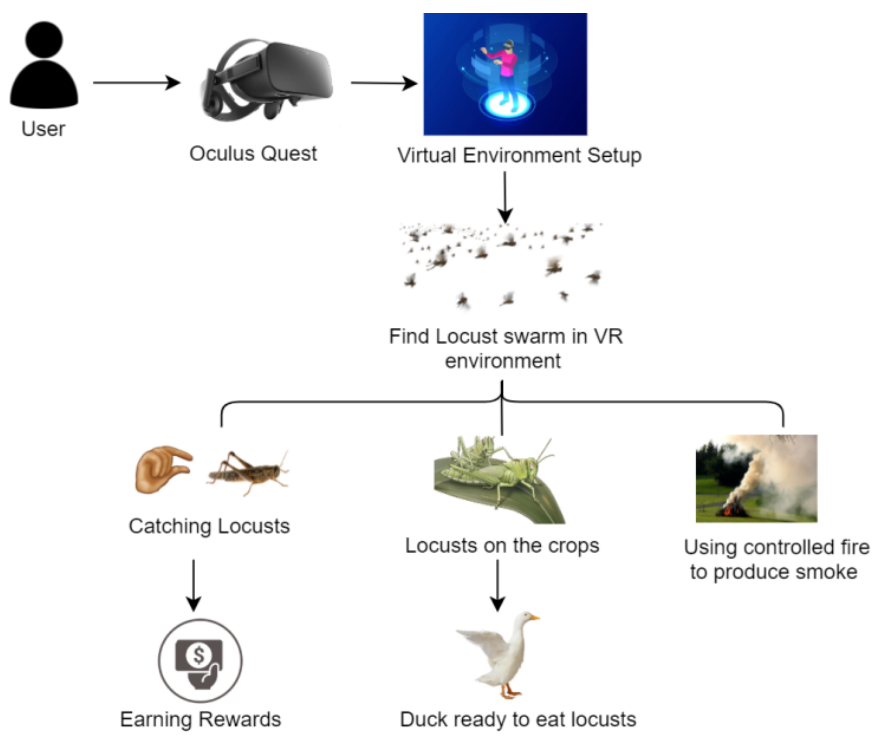

Fig. 4. Game Flow Diagram

\section{A. Game Story Flow}

The player begins by waking up and answering a phone call by touching the phone with their hand. They hear Natalina comment that she has just heard a swarm of locusts are coming to her farm. As they listen to Natalina describe what is about to happen, the player can read from a collection of facts related to sustainable locust control methods that are displayed in front of them.

After the phone call is finished, they pinch a door handle to go outside to their farm. This action shows the player how to use the pinch action, which is important for gathering locusts. 
Once the player is outside, there are already some locusts on the crops. In this tutorial section, the player can take as much time as they need to learn how to hit locusts with their hands and use the pinch action to collect them. If they do not perform the correct actions, Natalina reminds them what they need to do in order to proceed. After the player completes the tutorial, the first real stage begins.

In the first stage, locusts spawn randomly in groups coming from all four directions around the player. If a locust lands on a crop, it will begin eating it. If the player does not pinch the locust to catch it and earn money or shoo it away by hitting the locust with their hands, the locust will eventually eat the crop and the crop will become visibly skinnier. The crops do not replenish across stages, and if all crops are eaten by the locusts the player loses the game.

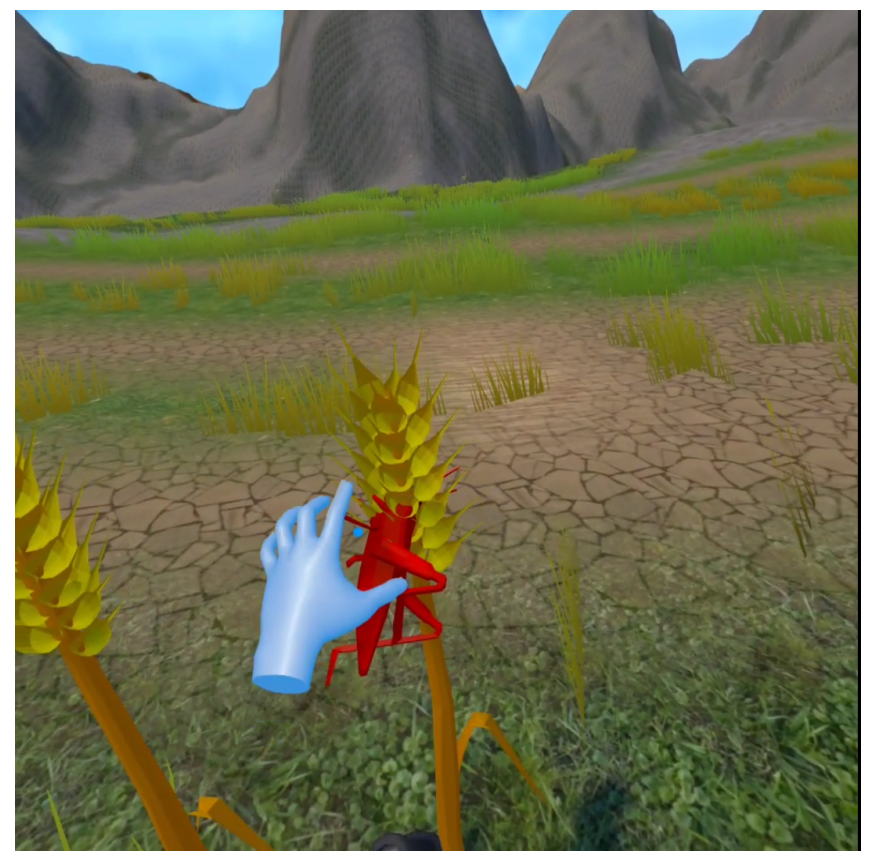

Fig. 5. Player trying to catch a locust

In the second stage, the player unlocks the ability to use smoke. They can activate the smoke by touching one of two piles of rubbish at their feet. Smoke causes the locusts to avoid going near the crops for a limited period of time. After the rubbish pile has burned up the smoke stops and the rubbish pile takes some time to recharge and become usable again.

In the third stage, the player unlocks the ability to use money to buy ducks. The player earns enough money to buy one duck after catching fifteen locusts, and they buy the duck by touching a pedestal with a duck on it located at their feet. Ducks will eat locusts, but for every locust a duck eats the player cannot catch the locust to earn more money, so the player needs to make sure they have enough locusts to catch to continue to earn money.

In the fourth and fifth stages, the player must handle increasingly large groups of locusts by catching locusts, using smoke strategically when there are too many locusts to handle

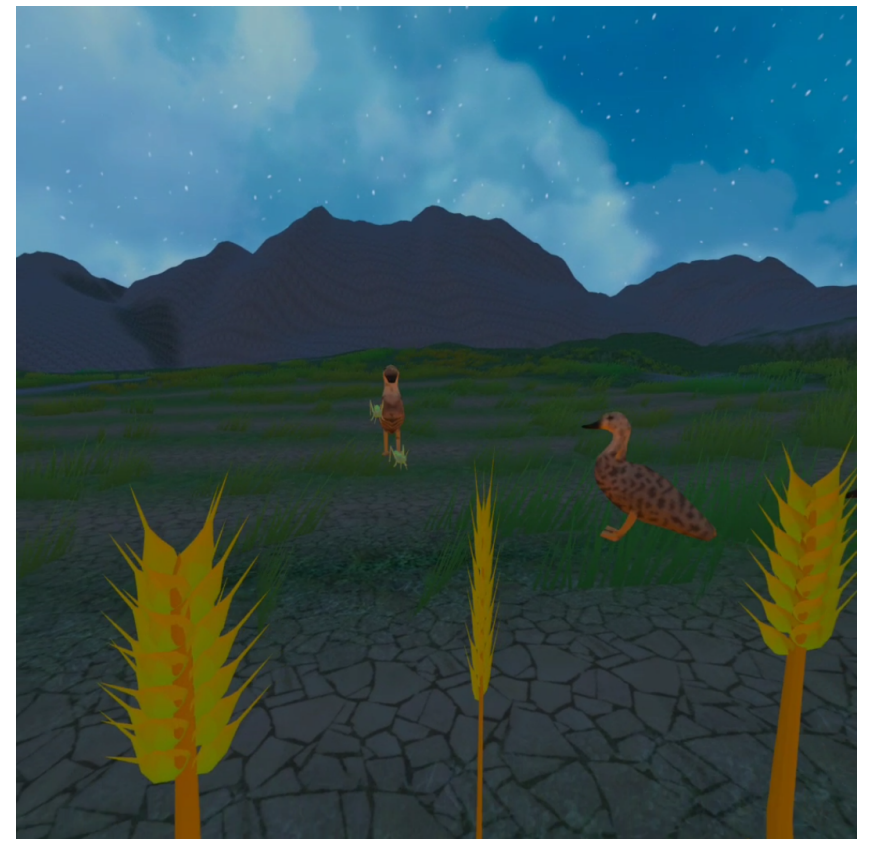

Fig. 6. Ducks eating locusts

at once, and buying more ducks which help to deal with the increasing swarm. There are three different win states depending on the amount of crops saved, which provides replayability for the experience.

\section{B. Game Mechanics and Systems}

This game makes use of the Oculus Quest headset's handtracking functionality. The player has two ways of interacting with objects in the world, touching an object with their hands or performing a pinch action with their thumb and index finger near an object. The player catches locusts by performing a pinch action, and shoos locusts away, activates smoke, and buys ducks by using a touch action.

We also looked at other ways of increasing player immersion. In VR, it takes effort for the player to turn their head, so we set up an audio system that would allow the user to know where locusts were coming from. The sounds of locusts flying and eating crops use spatialized audio, so that depending on where the user's head is facing they know what direction the locusts are coming from. For Natalina's narration audio, we used non-spatialized audio to give the player the impression that Natalina was speaking to herself in her head. There are also many audio clips of Natalina providing feedback to the player on key game events, such as when the player activates smoke or a locust eats a crop. This allows the player to know something important happened in the game even if it is not directly visible.

To make the locusts feel more realistic, we implemented a boid system in the game. This system causes the locust objects to bunch together to form swarms and fly with indirect patterns that make it harder for the player to predict their movements.

Lastly, to give the player an indication of progress of the game over time, we implemented a day and night cycle system. 


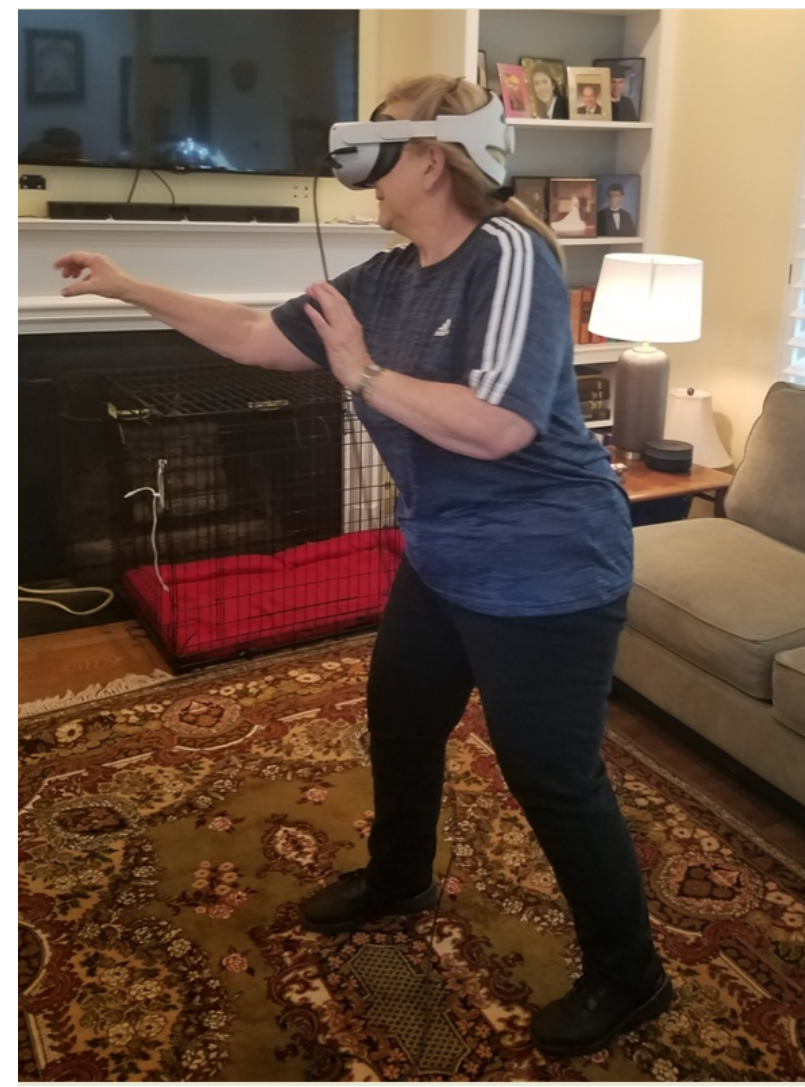

Fig. 7. User playing the game

The day begins in the morning, and the light changes over a period of time to reflect the sun coming up over the horizon. Over the course of the day, the sun reaches the peak and then gradually begins to go back down, ending with the player at night seeing the stars come out. This way, the player can use the position of the sun to determine how far into the game they have progressed.

\section{UsABILITy TESTING}

To refine the concept, mechanics, and difficulty curve of the game, a subjective evaluation performed. In both sets of tests, there was one experienced and one inexperienced VR user. In the early test, the experienced VR user became disoriented after a short time in the experience. Neither user could win the game after multiple attempts, so we made the game easier by reducing the number of locusts in the swarms and increasing the locust-eating efficiency of the ducks. In the next test, the inexperienced user commented that the omnidirectional nature of the design was slightly disorienting, but the experienced user was able to complete the game and win with the ending where they saved the greatest amount of crops.

Our testing indicates that future updates should offer options to the player to make the game easier, such as having the locusts only spawn from one direction or to reduce the amount of locusts if the player is having difficulty playing the full experience. In addition, we need to provide game instruction reinforcement with subtitles to help players learn how to play the game more quickly.

\section{CONCLUSION}

Climate change and extreme weather conditions are causing a rise in locust numbers because hot and humid weather conditions are favouring their breeding. Locust swarms are worsening food security issues and recently have become a threat for many countries. There is a need to use effective control measures without overusing pesticides which can cause long-term impacts to the environment. The objective of the game is to explain and teach the sustainable ways of controlling the locusts and saving the crops as well as explaining how farmers also use locusts as a source of income.

All of the methods of dealing with locusts shown in the game have allow farmers to handle locust swarms without resorting to pesticides and are more close to nature. The use of virtual reality and especially controller-free hand interaction in virtual space gives players an interactive and engaging way of learning about these locust control methods. The further work to this concept will add a new value to use of virtual reality as learning technology for sustainability.

\section{REFERENCES}

[1] S. Devi, "Locust swarms in east africa could be "a catastrophe"," The Lancet, vol. 395, no. 10224, p. 547, 2020.

[2] A. Sharma, "Locust control management: moving from traditional to new technologies-an empirical analysis," Entomol. Ornithol. Herpetol, vol. 4, no. 141, pp. 2161-0983, 2014.

[3] H. Bag and L. Bhoi, "Desert locust and climate change: A risk for agriculture," Biotica Research Today, vol. 2, no. 8, pp. 802-804, 2020.

[4] T. Yamano, N. Sato, and B. W. Arif, "Impact of covid-19 and locust swarms on farm households in sindh, pakistan: Analysis of data from a cross-sectional survey," 2020.

[5] W. Peng, N. L. Ma, D. Zhang, Q. Zhou, X. Yue, S. C. Khoo, H. Yang, R. Guan, H. Chen, X. Zhang et al., "A review of historical and recent locust outbreaks: Links to global warming, food security and mitigation strategies," Environmental research, vol. 191, p. 110046, 2020.

[6] L. Zhang, M. Lecoq, A. Latchininsky, and D. Hunter, "Locust and grasshopper management," Annual review of entomology, vol. 64, pp. 15-34, 2019.

[7] S. Krall, R. Peveling, and B. Diallo, New strategies in locust control. Birkhäuser, 2012.

[8] A. T. Showler, "A summary of control strategies for the desert locust, schistocerca gregaria (forskål)," Agriculture, ecosystems \& environment, vol. 90, no. 1, pp. 97-103, 2002.

[9] N. HC and J. R. Munavalli, "Automated real-time locust management using artificial intelligence."

[10] A. Auderer, "Kenya: Combating the growing locust swarms."

[11] K. Cressman, A. Van der Elstraeten, and C. Pedrick, "elocust3: An innovative tool for crop pest control," 2016.

[12] G. A. Matthews, "New technology for desert locust control," Agronomy, vol. 11, no. 6, p. 1052, 2021.

[13] M. A. Alalawi, "Desert locust control management: Innovative technology on the front lines in the battle against locust," in 2020 IEEE International Symposium on Safety, Security, and Rescue Robotics (SSRR). IEEE, 2020, pp. 4-4.

[14] X. Yao, D. Zhu, W. Yun, F. Peng, and L. Li, "A webgis-based decision support system for locust prevention and control in china," Computers and Electronics in Agriculture, vol. 140, pp. 148-158, 2017.

[15] C. Harmata. China is exploring sending 100,000 hungry ducks to battle pakistan's locust infestation. [Online]. Available: https://people.com/pets/china-sending-ducks-pakistan-locusts/

[16] M. Z. Iqbal and A. G. Campbell, "Investigating challenges and opportunities of the touchless hand interaction and machine learning agents to support kinesthetic learning in augmented reality," in 26th International Conference on Intelligent User Interfaces, 2021, pp. 99-101. 